\title{
El conocimiento como sistema adaptativo complejo en las organizaciones de gestión de proyectos (PMO)
}

Fecha de recepción: 9 de septiembre de 2016 Fecha de aprobación: 9 de diciembre de 2016 Pp. 27-54
Jeferson Eleazar Martinez Lozano1 Instituto Tecnológico Metropolitano jefersonmartinez@yahoo.es

DOI: https://doi.org/10.21158/23823399.v4.n2.2016.1626

Cómo citar este artículo/ To reference this article/Comment citer cet article/Para citar este artigo: Martínez, J. (2016). El conocimiento como sistema adaptativo complejo en las organizaciones de gestión de proyectos (PMO). Revista Ontare, 4(2), 27-54. DOI: https://doi.org/10.21158/23823399. v4.n2.2016.1626

\section{Resumen}

El artículo busca identificar la creación de conocimiento en sistemas adaptativos complejos (CAS) en las organizaciones de gestión de proyectos (PMO). Para tal fin, se revisaron resultados de investigaciones publicadas en revistas académicas, que han hecho un aporte comparativo de la conceptualización y caracterización de los CAS en las organizaciones. A partir de ahí, se plantea la hipótesis acerca de entender la importancia del conocimiento como CAS en las PMO y entender su relación con los modelos de madurez, como una herramienta de gestión de las capacidades en entornos multiagentes que interactúan en ambientes no lineales como sistemas complejos.

\section{Palabras clave}

Sistemas adaptativos complejos (CAS), oficinas de gestión de proyectos (PMO), conocimiento, modelos de madurez, gestión de proyectos.

\footnotetext{
${ }^{1}$ Ingeniero Telemático con experiencia en Dirección y Gestión de Proyectos. Magister en Sistemas de información por la Universidad EAFIT y especialista en proyectos informáticos por la Universidad Distrital de Bogotá. ORCID: http://orcid.org/0000-0003-4743-3500
} 


\title{
Knowledge as a complex adaptive system in project management organizations - (PMO)
}

\begin{abstract}
The article seeks to identify the creation of knowledge in complex adaptive systems (CAS) within project management organizations (PMOs). To that end, we reviewed the results of some research work that was published in academic journals, which have made a comparative contribution of the conceptualization and characterization of CAS in organizations. From there, we present the hypothesis about understanding the importance of knowledge as CAS in PMOs and understanding its relation with maturity models, as a tool for managing capacities in multi-agent environments that interact as complex systems in non-linear environments.
\end{abstract}

\section{Keywords}

Complex adaptive systems (CAS), project management offices (PMO), knowledge, maturity models, project management.

\section{La connaissance en tant que système adaptatif complexe des entreprises de gestion de projets - (PMO) \\ Résumé}

Cet article tente d'identifier la création de connaissance dans les systèmes adaptatifs complexes (CAS) des entreprises de gestion de projets (PMO). Nous avons pour cela procédé à une analyse des résultats de recherches publiés dans des revues académiques, ayant effectué un apport comparatif de la conceptualisation et caractérisation des CAS dans les organisations. Se pose dès lors l'hypothèse de l'importance de la compréhension de la connaissance des CAS dans les PMO pour comprendre leur relation avec les modèles de maturité comme outil de gestion des capacités en environnements multiagents interagissant dans des environnements non linéaires comme les systèmes complexes.

\section{Mots clefs}

Systèmes adaptatifs complexes (CAS), bureau de gestion de projets (PMO), connaissances, modèles de maturité, gestion de projets. 


\section{O conhecimento como sistema adaptativo complexo nas organizações de gerenciamento de projetos - (PMO)}

\section{Resumo}

$O$ artigo procura identificar a criação de conhecimento em sistemas adaptativos complexos (CAS) nas organizações de gestão de projetos (PMO). Para tal fim, revisaram-se resultados de pesquisas publicadas em revistas acadêmicas, com uma contribuição comparativa do conceito e da caracterização dos CAS nas organizações. A partir daí, propõe-se a hipótese do entendimento da importância do conhecimento como CAS nas PMO e entender sua relação com os modelos de maturidade como uma ferramenta de gestão das capacidades em meios multiagentes que interagem em ambientes não lineares como sistemas complexos.

\section{Palavras-chave}

Sistemas adaptativos complexos (CAS), escritórios de gestão de projetos (PMO), conhecimento, modelos de maturidade, gerenciamento de projetos. 


\section{Introducción}

T a importancia de la generación del conocimiento a partir de los Usistemas adaptativos complejos es un factor clave para el éxito de los proyectos, en donde entender las dinámicas y la complejidad de las interacciones entre múltiples agentes (organizaciones, procesos, metodologías, personas, herramientas) se vuelve fundamental a la hora de entregar resultados eficientes y eficaces a la gerencia en ambientes de sistemas complejos y no lineales. La oficina de gestión de proyectos (PMO) es la unidad funcional en las organizaciones encargada de brindar un acompañamiento y soporte a los gerentes de proyectos, para ayudar alcanzar dicho éxito la PMO aplica herramientas, modelos de madurez, plantillas, metodologías y técnicas de buenas prácticas que aseguren la gestión a través de la autoevaluación de las capacidades en las áreas de conocimiento correspondientes a la gestión de los proyectos.

Pese a la existencia de las PMO, la realidad sigue siendo desalentadora (figura1).

Figura 1. Estadísticas del resultado de los proyectos.

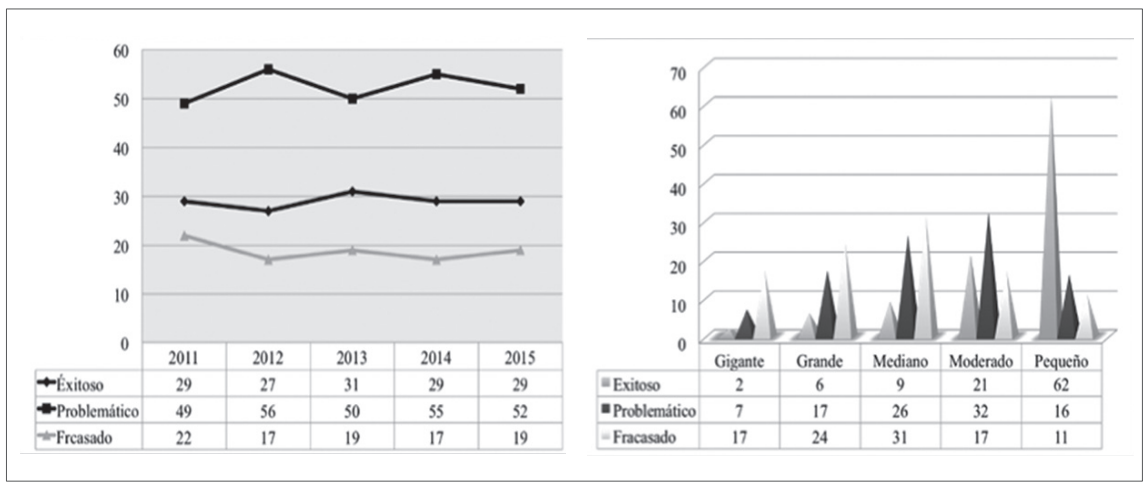

Fuente. Standish Group, 2015. 
Se puede observar en la figura 1 que la mayoría de los proyectos presentan problemas y están muy distantes del éxito y más cercanos al fracaso. Luego surgen los siguientes interrogantes: 1) ¿Cuál es la importancia de la generación de conocimiento como CAS en las oficinas de gestión de proyectos(PMO)?, y 2) ¿Por qué con todo el conocimiento alrededor del tema, y teniendo en cuenta que existen cada vez más personas capacitadas en gestión de proyectos, las PMO siguen teniendo dificultades en entregar los resultados esperados por la gerencia?

El presente documento tiene como alcance identificar la existencia y relación de la generación del conocimiento como sistema adaptativo complejo en las organizaciones de gestión proyectos. En la primera parte se hace una revisión de la literatura académica publicada por grupos de investigación y autores reconocidos en la temática, en donde se presenta un marco conceptual acerca de la definición y las características de los CAS, el concepto de conocimiento de los CAS, qué son las PMO y los modelos de madurez. En la segunda parte se busca establecer la conexión o relación entre la generación de conocimiento con las organizaciones como sistemas adaptativos complejos específicamente en las PMO. En seguida, se define una asociación del conocimiento generado por nivel a partir de la conceptualización del modelo de madurez, en una PMO que se comporta como CAS, como un modelo de madurez aplicado a la gestión de los proyectos, y la generación de conocimiento desde la dinámica de los CAS. Finalmente, se busca responder los interrogantes de la investigación y se entregan unas conclusionesde esta. 


\section{Fundamentos teóricos}

\subsection{Los sistemas adaptativos complejos (CAS)}

Para Ahmed, Elgazzar, \& Hegazi (2005) los sistemas adaptativos complejos son agentes adaptativos no homogéneos que pueden interactuar. En donde adaptativo se refiere a la capacidad de aprender.

Una propiedad emergente de los CAS es entender y estudiar sus agentes como un conjunto y no de forma individual. Algunos ejemplos de CAS son el cerebro, los sistemas sociales, la economía. Los sistemas adaptativos complejos se asocian con los siguientes conceptos: teoría del caos (Tetenbaum, 1998), teoría de la complejidad (Smith, 2005), ciencias de la complejidad (Kelly \& Allison, 1999) (Stacey R. , 2003) y el pensamiento sistémico (Senge, 1990) (Ackoff, 1999).

Los CAS se pueden modelar a partir de:

a. Ecuaciones diferenciales ordinarias (ODE) y ecuaciones diferenciales parciales (PDE).

b. Autómatas Celulares (AC) (Ilachinski, 2001).

c. Teoría evolutiva de juegos (Hofbauer \& Sigmund, 1998).

d. Los modelos basados en agentes.

e. Las redes (Watts \& Strogatz, 1998).

f. Cálculo fraccional (Stanislavsky, 2000).

\subsubsection{Las características de los sistemas adaptativos complejos}

De acuerdo con Rouse (2000), los CAS tienen las siguientes características:

- Son no lineales y dinámicos, como resultado los comportamientos del sistema pueden ser aleatorios o caóticos. 
- Se componen de agentes independientes cuyo comportamiento está basado en leyes físicas, psicológicas, sociales o de acuerdo con las exigencias de la dinámica del sistema.

- Dado que las necesidades o deseos de los agentes, que se refleja en sus reglas, no son homogéneos, sus objetivos y comportamientos son propensos a conflictos. En respuesta a estos conflictos o competiciones, los agentes tienden a adaptarse a los comportamientos de los demás.

- Los agentes son inteligentes. A medida que experimentan y adquieren experiencia, los agentes aprenden y cambian sus comportamientos en consecuencia. De este modo, el comportamiento global del sistema cambia con el tiempo.

- La adaptación y el aprendizaje tienden a dar lugar a la autoorganización. Patrones de comportamiento surgen en lugar de ser diseñado en el sistema. La naturaleza de los comportamientos emergentes puede variar de innovaciones valiosas a accidentes desafortunados.

- No hay un único punto (s) de control. Los comportamientos del sistema son a menudo imprevisibles e incontrolables, y nadie está a cargo. En consecuencia, los comportamientos de los sistemas adaptativos complejos, por lo general, pueden ser influenciados más fácilmente que controlado.

«Las organizaciones empresariales como CAS se caracterizan por la autoorganización y el surgimiento de un nuevo orden, para lo que es necesario el intercambio de información con el entorno, lo que las constituye en estructuras disipativas alejadas del equilibrio» (Nicolis \& Prigogine, 2007).

Para Stacey (1995), Anderson (1999), Mitleton (2003) y Holland (1992), la relación entre las características, propiedades y principios de los CAS como elemento de estudio de las organizaciones se resume en la tabla 1: 
Tabla 1. Relación entre las características y propiedades de los CAS.

\begin{tabular}{|c|c|c|c|c|}
\hline Stacey (1995) & $\begin{array}{c}\text { Anderson } \\
\text { (1999) }\end{array}$ & Mitleton (2003) & \multicolumn{2}{|c|}{ Holland (1992) } \\
\hline $\begin{array}{c}\text { Autoorganización } \\
\text { y emergencia de } \\
\text { orden }\end{array}$ & $\begin{array}{l}\text { El sistema se } \\
\text { autoorganiza } \\
\text { a partir de las } \\
\text { interacciones } \\
\text { emergentes entre } \\
\text { los agentes. Los } \\
\text { agentes que } \\
\text { conforman el } \\
\text { sistema siguen } \\
\text { diferentes } \\
\text { esquemas o } \\
\text { estructuras de } \\
\text { conocimiento } \\
\text { que determinan } \\
\text { la acción que } \\
\text { toma el agente } \\
\text { en un periodo de } \\
\text { tiempo. }\end{array}$ & $\begin{array}{l}\text { Emergencia del } \\
\text { orden a partir } \\
\text { de procesos de } \\
\text { autoorganización. } \\
\text { Intercambio de } \\
\text { información con } \\
\text { el entorno, lo cual } \\
\text { mantiene a las } \\
\text { organizaciones } \\
\text { como sistemas } \\
\text { alejados del } \\
\text { equilibrio al crear } \\
\text { nuevas estructuras } \\
\text { y orden. }\end{array}$ & $\begin{array}{c}\text { Modelos } \\
\text { internos } \\
\text { Flujos } \\
\text { marbetes }\end{array}$ & $\begin{array}{c}\text { Auto- } \\
\text { organización } \\
\text { y emergencia }\end{array}$ \\
\hline No linealidad & $\begin{array}{l}\text { Los agentes } \\
\text { coevolucionan } \\
\text { uno a otros, cada } \\
\text { agente se adapta } \\
\text { a su ambiente } \\
\text { esforzándose } \\
\text { por ajustarse } \\
\text { o adaptarse a } \\
\text { una función en } \\
\text { el tiempo; el } \\
\text { ajuste individual } \\
\text { depende de la } \\
\text { escogencia que } \\
\text { otros agentes } \\
\text { han hecho. } \\
\text { Nuevos } \\
\text { agentes pueden } \\
\text { formarse por la } \\
\text { recombinación } \\
\text { de elementos } \\
\text { previamente } \\
\text { exitosos. }\end{array}$ & $\begin{array}{l}\text { Exploración } \\
\text { del espacio de } \\
\text { posibilidades y } \\
\text { generación de } \\
\text { variedad como } \\
\text { condición para la } \\
\text { supervivencia. } \\
\text { Coevolución de } \\
\text { los sistemas, la } \\
\text { cual se presenta } \\
\text { en la medida en } \\
\text { que un elemento } \\
\text { influye y, a la vez, } \\
\text { es influenciado } \\
\text { por los demás. } \\
\text { Interrelación, } \\
\text { interacción e } \\
\text { interconectividad } \\
\text { de los elementos } \\
\text { del sistema y } \\
\text { entre este con el } \\
\text { ambiente. }\end{array}$ & $\begin{array}{l}\text { Diversidad } \\
\text { No linealidad } \\
\text { Agregación } \\
\text { Bloques de } \\
\text { construcción }\end{array}$ & $\begin{array}{l}\text { Evolución y } \\
\text { coevolución }\end{array}$ \\
\hline
\end{tabular}

Fuente. Elaboración propia. 
El comportamiento de las organizaciones como CAS se encuentra soportado en los trabajos de autores como: Nicolis \& Lefever (1975), Prigogine (1983), Kauffman (1995), Gellman (1994 y 1995) y Holland (1995).

\subsection{Qué es una oficina de dirección de proyectos (PMO)}

De acuerdo con el PMI ${ }^{\circledR 2}$ :

Una oficina de dirección de proyectos es un cuerpo o entidad dentro de una organización que tiene varias responsabilidades asignadas con relación a la dirección centralizada y coordinada de aquellos proyectos que se encuentran bajo su jurisdicción. Las responsabilidades de una oficina de dirección de proyectos pueden abarcar desde el suministro de funciones de soporte para la dirección de proyectos hasta la responsabilidad de la dirección directa de un proyecto (PMI, 2008).

Desde un enfoque académico, Hobbs (2007) realizó un estudio aplicado a 500 PMO a nivel global, donde se les preguntó acerca de las funciones que debe desempeñar una PMO (tabla 2).

2 El Project Management Institute es una organización internacional sin fines de lucro que asocia a profesionales relacionados con la Gestión de proyectos. 
Tabla 2. Funciones de la PMO

\begin{tabular}{|c|c|}
\hline Funciones de la PMO & $\begin{array}{c}\% \text { de } \\
\text { importancia }\end{array}$ \\
\hline Informe del estado del proyecto a la alta dirección & $83 \%$ \\
\hline Desarrollar e implementar una metodología estándar & $76 \%$ \\
\hline Monitorear y controlar el rendimiento de los proyectos & $65 \%$ \\
\hline Desarrollo de competencias del personal, incluida las capacitaciones & $65 \%$ \\
\hline Implementar y operar un sistema de información del proyecto & $60 \%$ \\
\hline Prestar asesoría a la alta dirección & $60 \%$ \\
\hline Coordinación entre proyectos & $59 \%$ \\
\hline Desarrollar y mantener un cuadro de indicadores del proyecto & $58 \%$ \\
\hline Promover la gestión de proyectos en la organización & $55 \%$ \\
\hline Controlar y monitorear el desempeño de la PMO & $50 \%$ \\
\hline Participar en la planificación estratégica de la organización & $49 \%$ \\
\hline Proveer mentores a los directores de proyectos & $49 \%$ \\
\hline Administrar uno o más portafolios & $49 \%$ \\
\hline Identificar, seleccionar y priorizar nuevos proyectos & $48 \%$ \\
\hline Administrar los archivos de documentación del proyecto & $48 \%$ \\
\hline Administrar uno o más programas & $48 \%$ \\
\hline Auditoría de los proyectos & $45 \%$ \\
\hline Administrar interfaces del cliente & $45 \%$ \\
\hline $\begin{array}{l}\text { Proporcionar un conjunto de herramientas y sin un esfuerzo para } \\
\text { estandarizar }\end{array}$ & $42 \%$ \\
\hline Ejecutar tareas especializadas para administradores de proyectos & $42 \%$ \\
\hline Asignar recursos entre proyectos & $40 \%$ \\
\hline Llevar a cabo revisiones posteriores al proyecto & $38 \%$ \\
\hline Implementar y administrar la base de datos de lecciones aprendidas & $34 \%$ \\
\hline Implementar y administrar bases de datos de riesgo & $29 \%$ \\
\hline Gestión de los beneficios & $28 \%$ \\
\hline Llevar a cabo la creación de redes y el análisis ambiental & $25 \%$ \\
\hline $\begin{array}{l}\text { Reclutar, seleccionar, evaluar y determinar los sueldos de los } \\
\text { directores de proyectos }\end{array}$ & $22 \%$ \\
\hline
\end{tabular}

Fuente. Elaboración propia. 
El concepto de organización de proyectos ha surgido recientemente y es definido como: «La parte del negocio que se relaciona directa o indirectamente con proyectos, con el propósito de lograr los objetivos de una empresa o varias empresas»' (Artto \& Wikstrom, 2005).

\subsection{Qué son los modelos de madurez}

Un modelo de madurez (figura 2) es un conjunto estructurado de elementos (buenas prácticas, herramientas de medición, criterios de análisis, etc.) que permite identificar las capacidades instaladas en dirección de proyectos en la organización, compararlas con estándares, identificar vacíos o debilidades y establecer procesos de mejora continua (Claros, 2015).

Figura 2. Capacidades de madurez de la PMO.

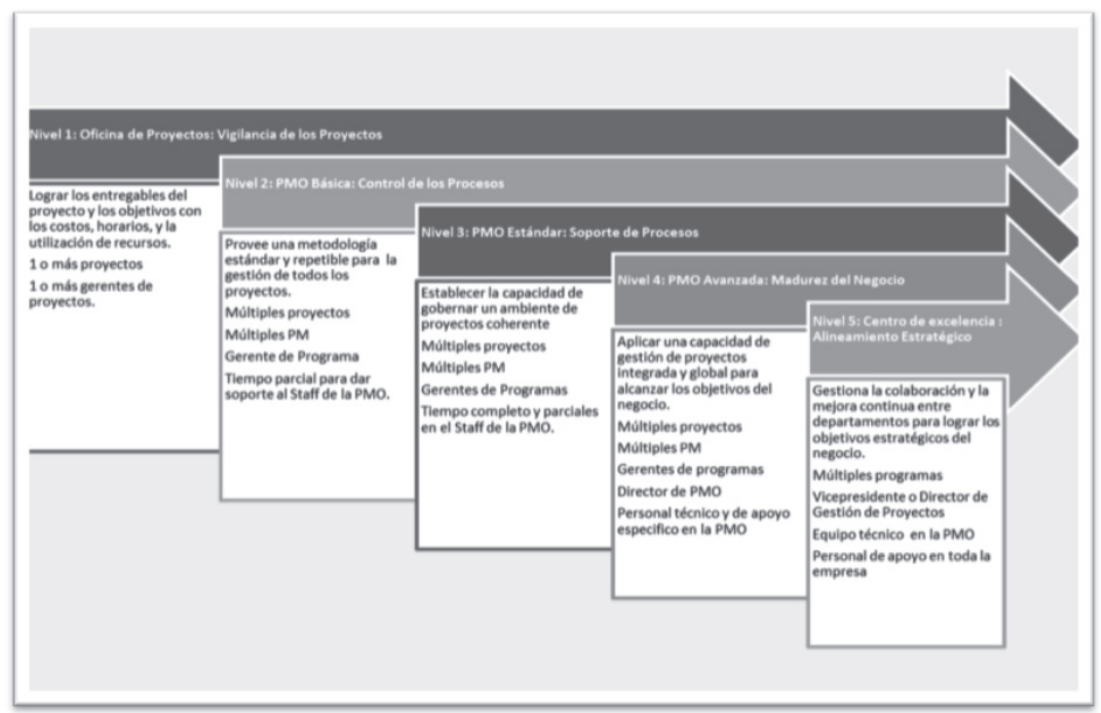

Fuente. Elaboración propia.

De acuerdo con el CBP, con un nivel de madurez en grado 5 una PMO puede mejorar sus capacidades de desempeño en la organización hasta en un $50 \%$ (PM Solutions, 2007). 


\section{Metodología}

Dara el estudio se consideró la revisión de la literatura académica 1 existente, a través de plataformas como Elsevier, IEEE y ScienceDirect entre otras, considerando que las publicaciones han desempeñado un rol importante en la temática de los sistemas adaptativos complejos y la generación de conocimiento en las organizaciones de gestión de proyectos.

A partir de la revisión de las diferentes fuentes, el autor realiza un análisis que ayuda a relacionar la existencia de la creación del conocimiento como sistema adaptativo complejo (CAS) en las oficinas de gestión de proyectos (PMO), al igual que buscar visualizar las tendencias investigativas en el campo.

\section{Desarrollo del trabajo}

\subsection{Definición de conocimiento desde los sistemas adaptativos complejos}

Un sistema adaptativo complejo es un término creado por Holland (1975) para describir:

Sistemas no lineales cuyo comportamiento está determinado por la interacción de sus partes adaptativas. Las numerosas partes que componen la estructura y el nivel de interacción que existe entre las partes son las principales características distintivas de un sistema complejo. 
El concepto de sistemas adaptativos complejos fue introducido por el Instituto de Santa Fe, en un intento de proporcionar una nueva perspectiva a la dinámica de los sistemas complejos. Un CAS se compone de muchos agentes que interactúan de diferentes maneras y capacidad (Cowan \& Pines, 1994).

De acuerdo con Andrew y Cynthia (2009), el concepto de conocimiento desde el enfoque de los sistemas adaptativos complejos se puede entender de dos formas: «(tácito y explícito) o desde una dimensión ontológica (individuo, grupos, organización)» (T \& P, 2009, p. 218). El conocimiento tácito es utilizado principalmente a través de interacciones sociales (Hansen, 2002, p. 232), (NonakaI, 1991, p. 96), y en las organizaciones de gestión de proyectos se fundamenta en la experiencia de los profesionales; mientras que el conocimiento explícito puede ser codificado, capturado y difundido por vía electrónica (W \& C, 2005, p. 731), y se refleja en la PMO en la documentación de las lecciones aprendidas, las buenas prácticas utilizadas y la sistematización de estas para ayudar a la toma de decisiones y resolución de problemas de forma eficiente y eficaz.

En este sentido, de acuerdo con Rivas (2014):

Entender el conocimiento desde una perspectiva compleja implica comprenderlo no solo en subjetividad sino en contexto. Este contexto alude no solo a los aspectos culturales en los que se sitúa el sujeto que conoce, sino a la temporalidad en la que sujeto y objeto se relacionan.() ...es una propiedad de los agentes que están dispuestos entre sí para actuar en circunstancias particulares (p. 86).

Mientras que para Boisot (1998) el conocimiento desde una perspectiva compleja es como «una capacidad que se construye en información que ha sido extraída de datos. Los activos de conocimiento producen una corriente de servicios útiles a lo largo del tiempo y, por lo tanto, tienen un valor económico potencial» (p. 109). 


\subsection{Creación de conocimiento en los sistemas adaptativos complejos}

La creación de conocimiento en los CAS generalmente implica tres fases: un mecanismo de introducción de variación al conocimiento, un proceso de selección consistente y un mecanismo para preservar y reproducir las variaciones seleccionadas (Campbell, D., 1960, pp. 80-400). Dicho conocimiento solo se genera a partir de interacciones con múltiples agentes que pueden adaptarse al entorno de acuerdo con sus objetivos (Chen, 2007, págs. 2-4).

El proceso de creación de conocimiento a partir de los CAS en las organizaciones se rige por una serie de mecanismos que permiten a los agentes interactuar entre sí. Dichos mecanismos son: las relaciones, asignación de créditos, la explotación y exploración. Ver (figura 3).

Figura 3. Procesos de los CAS para crear conocimiento.

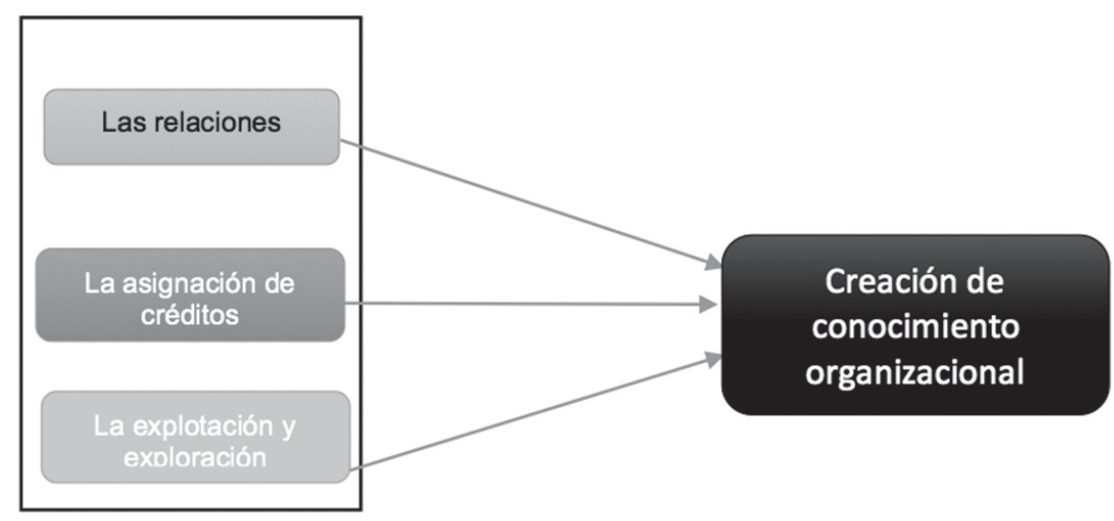

Fuente. Elaboración propia.

Las relaciones consideran que, durante su vida útil, una organización acumula un gran número de activos de conocimiento. La captura de cada experiencia puede no añadir ningún valor si el conocimiento no se traduce en un cambio de comportamiento para mejorar el rendimiento. Los 
créditos asignados se refieren a que la capacidad de las organizaciones para crear nuevos conocimientos, a partir de los activos existentes, requiere una evaluación rigurosa de su base de conocimientos. Mientras que la explotación y exploración son procesos que las organizaciones utilizan para resolver problemas. Una organización puede decidir sobre la conveniencia de solucionar un problema mediante la explotación de los conocimientos incorporados a sus activos de conocimiento prefabricados o mediante la exploración de nuevas ideas.

En este sentido Bohórquez (2013) dice que:

La generación de conocimiento e ideas innovadoras cuando un equipo está trabajando junto pueden describirse como una propiedad emergente en el sentido que surge de la interacción de los individuos, y no es solo la suma de ideas existentes (Mitleton, 2003); adicionalmente, ese conocimiento podría generar algo nuevo y sorprendente (Espinosa \& Porter, 2011). Es decir, la coevolución implica redes de relaciones no lineales entre los agentes, en donde las interacciones permanentemente cambiantes hacen imposible la predicción de estados futuros (Holland J. H., 1998) (Bohórquez, 2013).

\subsection{El conocimiento como CAS en las oficinas de gestión de proyectos}

Las organizaciones de gestión de proyectos, conocidas como PMO, son sistemas no lineales que demandan una constante reingeniería de los procesos, que les permita ser sensibles a los nuevos cambios a partir de la adaptación y el aprendizaje de múltiples agentes que interactúan entre sí. Dichos agentes pueden ser organizaciones, individuos, grupos, procesos, metodologías, tecnologías o, incluso, el conocimiento especializado, donde las organizaciones empresariales son sistemas 
caracterizados por ciclos de feedback no lineal, por cuanto todo el tiempo las personas interactúan unas con otras (Stacey D. R., 1995). Y la no linealidad asociada a las características CAS de: emergencia, la autoorganización y la evolución, está reflejada en que las decisiones $\mathrm{y}$ acciones de cualquiera de los agentes que forman parte de las PMO pueden o no afectar a los otros.

Los CAS en las organizaciones de gestión de proyectos se pueden asociar acorde con el agente y sus atributos con el que se esté interactuando. Por ejemplo, en la gestión de los proyectos se puede asociar a la complejidad presente en la gestión del alcance, la gestión de los costos, la gestión de los riesgos y el control del cambio.

Por ejemplo, en el control del cambio se pueden presentar modificaciones en la metodología, pasando de un enfoque agile a un enfoque tradicional por la dinámica del proyecto, lo que conlleva nuevas interacciones por parte de los agentes involucrados, y eso implica complejidad desde un enfoque de los CAS.

Otras de las características del PMBOK asociadas a la complejidad de los proyectos y sus atributos son:

- El número de fases, la necesidad de fases y el grado de control aplicada (PMI 2013, p. 41).

- El contenido del plan de gestión de proyectos (PMI 2013, p. 74).

- El tamaño de la carta del proyecto (PMI 2013, p. 74).

- El nivel aplicado de control de cambios (PMI 2013, p. 96).

- El nivel de detalle de los paquetes de trabajo (PMI 2013, p. 128).

- El costo y la exactitud de la estimación de costos de abajo hacia arriba (PMI 2013, p. 205).

- La necesidad de evaluaciones formales o informales de desempeño del proyecto (PMI 2013, p. 282).

- El número de grupos de interesados (PMI 2013, p. 394). 
Otros atributos asociados a la complejidad de los proyectos se pueden ver en la tabla 3:

Tabla 3. Atributos de complejidad en los proyectos.

\begin{tabular}{|l|l|}
\hline \multicolumn{1}{|c|}{ Otros atributos de complejidad } & \multicolumn{1}{c|}{ Referencia } \\
\hline \multirow{2}{*}{ Tamaño del proyecto } & Pich, Loch \& Meyer (2002) \\
\cline { 2 - 2 } & Thomas and Mengel (2008) \\
\hline \multirow{2}{*}{ Estilo de liderazgo } & Turner \& Müller (2006) \\
\cline { 2 - 2 } & Müller, Geraldi \& Turner (2007) \\
\hline Ambigüedad de tareas & Pigagaite, Silva \& Hussein (2013) \\
\hline Variaciones de perímetro & Pigagaite, Silva \& Hussein (2013) \\
\hline La complejidad interna de los elementos del proyecto & Müller, Geraldi \& Turner (2007) \\
\hline La falta de solidez de los elementos del proyecto & Ramasesh \& Browning (2014) \\
\hline
\end{tabular}

Fuente. Elaboración propia.

El conocimiento se debe crear, transferir y reutilizar de una forma inmersa en la metodología utilizada por los proyectos, y posteriormente se debe repetir el ciclo para que se convierta en explícito dentro de la gestión de los proyectos a través de la documentación acerca de las lecciones aprendidas (figura 4).

Figura 4. El conocimiento en los proyectos.

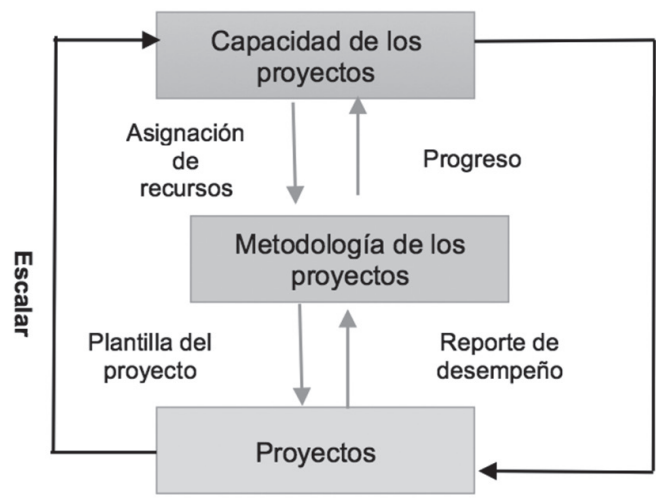

Resolución

Fuente. Elaboración del autor. 
Gudi y Becerra-Fernández (2006) estudiaron el papel del conocimiento en la gestión de proyectos en organizaciones como sistemas complejos, y se centraron en los aspectos dinámicos, y argumentan que, si somos capaces de comprender mejor la naturaleza de los sistemas de riesgo, podemos ser capaces de reducir o eliminar el fracaso y aumentar las posibilidades de éxito del proyecto. También consideran que: cuando aumenta la complejidad del proyecto, es importante encontrar los medios para gestionar la interrelación de los procesos, con las actividades y eventos en los ambientes del proyecto.

Mientras que para Perry (2012) las PMO se mueven entre cuatro cuadrantes (figura 5).

Figura 5. Los CAS en la gestión de proyectos.

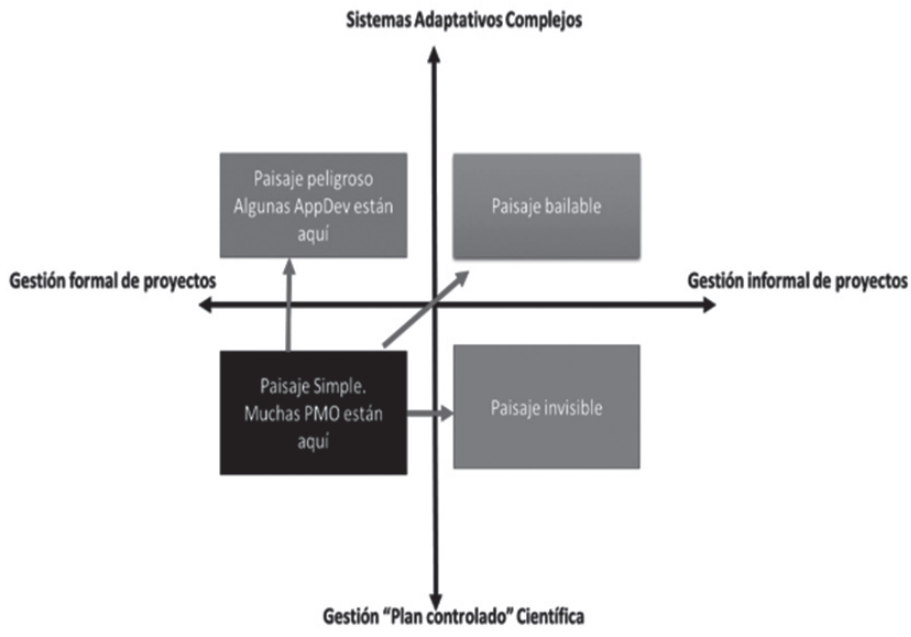

Fuente. Elaboración del autor.

En donde la mayoría de las PMO se ubican en el tercer cuadrante correspondiente a una gestión formal de los proyectos a través de una metodología estandarizada, junto con un plan controlado para los sucesos e imprevistos que se puedan presentar en cualquiera de las fases (inicio, planeación, control y cierre). 
Dichas PMO pueden pasar en cualquier momento a alguno de los otros cuadrantes por diferentes razones: 1) no cuentan con el apoyo de los directivos, 2) no tienen gerentes de proyectos con un entrenamiento y unas competencias en un estándar de gestión de proyectos, 3) poca claridad frente a los objetivos del proyecto, 4) un uso de herramientas tecnológicas no estandarizadas o simplemente por aspectos culturales, sociales o dinámicas organizaciones. Lo que obliga a las PMO a moverse en el plano emergente dentro del campo de los CAS y buscar apoyo por fuera de la organización en diferentes comunidades, redes sociales o agentes expertos en gestión de proyectos. Un ejemplo son las AppDev ${ }^{4}$.

La captura del conocimiento se puede ver representada en cualquiera de los cuatro cuadrantes de forma explícita, en el caso de tener una gestión de proyectos informal, o de forma tácita, si se sigue una dirección formal frente a la gestión de estos.

Para poder responder a las preguntas planteadas en la investigación: 1) ¿Cuál es la importancia de la generación de conocimiento como CAS en las organizaciones de gestión de proyectos?, y 2) ¿Por qué con todo el conocimiento alrededor del tema, y teniendo en cuenta que existen cada vez más personas capacitadas en gestión de proyectos, las PMO siguen teniendo dificultades en entregar los resultados esperados por la gerencia? Para responder dichos interrogantes es necesario relacionar los modelos de madurez con la dinámica de los sistemas adaptativos complejos porque, a partir de ahí, una organización puede empezar a reconocer sus capacidades de gestión en proyectos y llegar a capturar el conocimiento en cada uno de los diferentes niveles y luego gestionarlo. Ver figura 6).

4 Es una inmersión tecnológica, donde emprendimientos robustecen sus productos por medio del desarrollo de software, métricas de negocio y pruebas de usuario. 
Figura 6. Niveles del modelo de madurez.

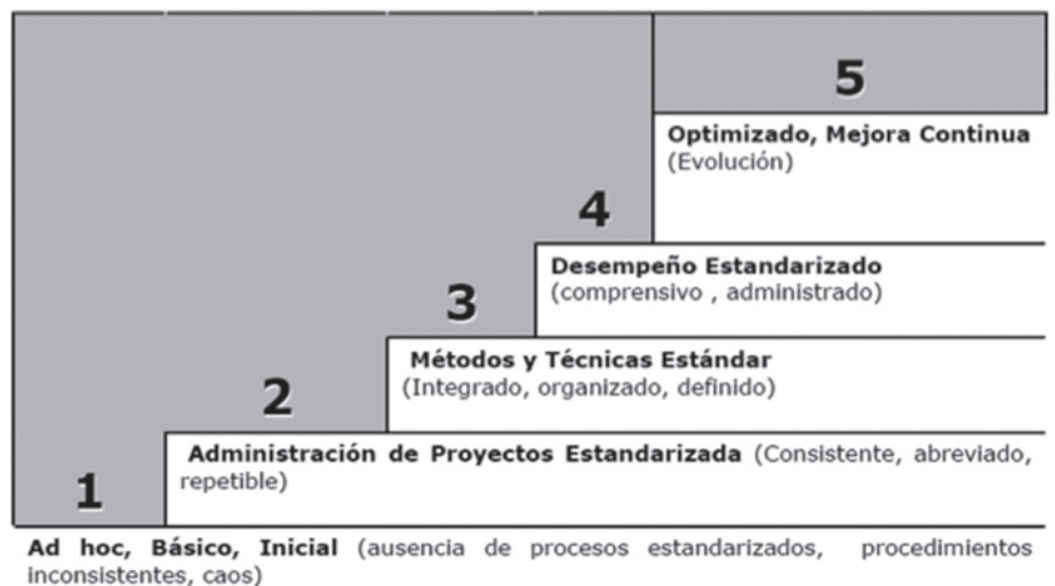

Fuente. Elaboración del autor.

En el nivel 1 del modelo de madurez, el grado de complejidad es bastante alto debido a la informalidad que se presenta en cada una de las etapas de gestión del proyecto, quedando la responsabilidad absoluta en el conocimiento adquirido y las habilidades gerenciales de cada director de proyectos (PM). Razón por la cual, las interacciones entre múltiples agentes que trabajen de forma conjunta, independientemente de los métodos aplicados y las herramientas utilizadas, es algo que no existe en este nivel. Luego, la creación de conocimiento desde un enfoque de las CAS en las organizaciones de gestión de proyectos se puede presentar a partir del esfuerzo de cada líder de proyecto y su interacción con otras personas o redes de aprendizaje, como internet o grupos académicos interdisciplinados establecidos.

Para el nivel 2 las organizaciones de gestión de proyectos ya han formalizado un estándar para gestionar los procesos, se comienzan a estabilizar los procedimientos de la organización, sus herramientas, y existe un interés por el aprendizaje grupal. La captura y generación del conocimiento como CAS en las organizaciones de gestión de los 
proyectos en este nivel sigue siendo una necesidad junto con un esfuerzo y disciplina más individual que colectivo dentro de la PMO.

En el nivel 3 comienza una integración formalizada basada en roles y con líderes de proyectos especializados en una metodología estandariza, también comienza a ver un trabajo colaborativo a través de una plataforma tecnológica entre los miembros del equipo del proyecto, junto con una ruta por seguir. La PMO vista desde los CAS va perdiendo el concepto de sistemas NO lineales en este nivel, y la generación de conocimiento se vuelve un desafío porque el conocimiento ya no se construye ni se captura desde diversas fuentes de saberes, sino desde un roadmap ya definido por la organización y líderes de proyectos que siguen un estándar de gestión claramente definido.

Para los niveles 4 y 5 existe una integración efectiva con un centro de gestión del trabajo por competencias, una apropiación y optimización de los factores de riesgos, gerentes de proyectos que siguen múltiples métodos de gestión, se empiezan a realizar una trazabilidad sobre los beneficios, se documentan las lecciones aprendidas y los proyectos comunican eficazmente a la gerencia. La PMO vista como CAS en estos niveles se convierte en un generador de conocimiento explícito basado en el aprendizaje y experiencia que se genera a partir de las lecciones y las buenas prácticas documentadas.

Dicha relación entre los niveles de madurez y la gestión de proyectos a través de las PMO como sistemas adaptativos complejos para la generación de conocimiento se resume en: para los niveles del 1 al 3 la PMO se comporta como un sistema no lineal, y las interacciones de los agentes para generar conocimiento queda bajo el esfuerzo individual de los coordinadores de proyectos que, a su vez, aplican un conocimiento tácito (basado en sus experiencias, redes sociales, grupos académicos interdisciplinados) como aprendizaje de las experiencias en el tiempo. 
Para los niveles 4 y 5 en las PMO verlas como CAS es difícil, porque todo está formalizado y estandarizado. Luego no sé comportan de una forma no lineal, pero existe: 1) captura del conocimiento, 2) creación del conocimiento, 3) transferencia de conocimiento y 4) reutilización del conocimiento, derivados en un conocimiento explícito documentado en el tiempo, junto con guías de buenas prácticas e indicadores de desempeño.

Además de los niveles de madurez, también es necesario entender el aprendizaje que se genera a partir de las interacciones entre los ambientes externos (proveedores, factores económicos, sociopolíticos, cultura, demografía, marco regulatorio), ambientes internos del proyecto (roles y responsabilidades, equipo del proyecto, conocimiento, habilidades, experiencias, inteligencia emocional, interacciones sociales, tipos de personalidades y liderazgo), producto (tecnología, hardware, software, aplicaciones, bases de datos, sistemas de información), clientes y proveedores, por qué pueden ser la esencia para la creación de un aprendizaje que conlleve conocimiento en las organizaciones a partir de los CAS.

A la pregunta: ¿Cuál es la importancia de la generación de conocimiento como CAS en las organizaciones de gestión de proyectos?

Que ayuda a mantener un buen desempeño y rendimiento para alcanzar el éxito en los resultados entregados en la gestión de los proyectos en ambientes complejos, que la organización de gestión de proyecto puede alcanzar niveles de innovación demasiado altos al trabajar en ambientes complejos y con múltiples agentes que interactúan entre sí, ayuda a eliminar o reducir los riesgos que se presentan en los proyectos de forma significativa $y$, finalmente, porque permite tener el equipo del proyecto cohesionado entorno a un mismo objetivo. Y, ¿por qué con todo el conocimiento del tema, y teniendo en cuenta que existen cada vez más personas capacitadas en gestión de proyectos, las PMO siguen teniendo dificultades en entregar los resultados esperados por la gerencia? 
La respuesta a esta pregunta puede estar en entender las dinámicas y transformaciones que pueden tener las organizaciones de gestión de proyectos en cualquier momento, en donde es necesario sufrir mutaciones sobre la marcha para poder adaptarse rápidamente a los ambientes complejos demandados y entregar resultados menos fallidos acordes con las estadísticas actuales, ya que en muchos escenarios no es visto como una gestión más allá de la gestión del cambio.

\section{Conclusiones}

1 término adaptativo es poco afortunado, dado que no necesariamente 1 puede ser entendido desde la evolución, sino que puede limitarse a la acomodación y a las condiciones cambiantes. En otras palabras, en el contexto de la administración el término es restringido y, por tanto, se sugiere usar la expresión de sistemas complejos, en consonancia con la organización de gestión de proyectos como sistema complejo.

Los principales procesos de gestión del conocimiento son: 1) captura del conocimiento, 2) creación del conocimiento, 3) transferencia de conocimiento y 4) reutilización del conocimiento. A partir de dichos procesos se puede establecer una conexión con la forma como se genera un aprendizaje en los niveles de madurez de la PMO en ambientes complejos no lineales, ya sea de forma tácita o explícita.

El concepto de CAS en el contexto de las PMO se centra en las interacciones de múltiples agentes representados en atributos vistos desde la complejidad y asociados a cada uno de los niveles de madurez en gestión de proyectos. 


\section{Referencias}

Ackoff,R.(1999). Re-creating the Corporation: A Design of Organizations for the 21st Century. New York, NY: Oxford University Press.

Ahmed, E., Elgazzar, A., \& Hegazi, A. (2005). An Overview of Complex Adaptive Systems. Recuperado de https://arxiv.org/pdf/nlin/0506059. pdf

Anderson, P. (1999). Complexity theory and organization science. Organization Science, 10(3), 216-232.

Artto, K., \& Wikstrom, K. (2005). What is project business? International Journal of Project Management, 23(5), 343-353.

Bohórquez, E. (2013). La organización empresarial como sistema adaptativo complejo. Estudios gerenciales, 29(127), 258-265.

Chen, X. (2007). Research on the Connotation and the Framework of Knowledge Management based on Complex Adaptive System. IEEE.

Claros, A. (Marzo de 2015). Projectools. Herramientas para la Gestión de Proyectos. Recuperado de https://projectools.wordpress.com/ modelos-de-madurez-en-gestion-de-proyectos

Cowan, G.; Pines, M. \& Meltzer, D. (Eds.) (1994). Complexity, metaphors, models, and reality: Westview Press.

Campbell, D.T. (1960). Variation and selective retention in creative thought as in other knowledge process. Psychiatric Review, 67, 380400 . 
Espinosa, A., \& Porter, T. (2011). Sustainability, complexity and learning: Insightsfrom complex systems approaches. Learning Organization, 18(1), 54-72.

Gudi, A., \& Becerra-Fernández, I. (2006). Role of knowledge management in project management of complex systems organizations. NASA. Knowledge Management and Succesful Mission Operations Conference, Houston, TX.

Hansen, M. (2002). Knowledge networks: explaining effective knowledge sharing in multiunit companies, Organization Science. 13(3), 232-248.

Hobbs, B. (2007). The Multi-Project PMO: A Global Analysis of the Current State of Practice. Quebec, Canada: University of Quebec at Montreal.

Hofbauer, J., \& Sigmund, K. (1998). Evolutionary games and population dynamics. Cambridge University Press.

Holland, J. (1995). Complex adaptive systems. Daedalus, 121(1), 17-30.

Holland, J. H. (1998). Emergence: From Chaos to Order. Perseus BooksGroup.

Ilachinski, A. (2001). Cellular Automata. A Discrete Universe. USA: Center for Naval Analyses.

Kauffman, S. (1995). At Home in the Universe: The Search for Laws of Self-Organization and Complexity. UK: Oxford University Press.

Kelly, S., \& Allison, M. (1999). The Complexity Advantage: How the Science of Complexity Can Help Your Business Achieve Peak Performance. New York, NY: McGraw-Hill. 
Mitleton, K. E. (2003). Ten principles of Complexity \& Enabling Infrastructures. InComplex Systems and Evolucionary Perspectives of Organisations: The Applicationof Complexity Theory to Organisations. UK: London Shool of Economics.

Nicolis, G., \& Lefever, R. (1975). Membranes, Dissipative Structures and Evolution. New York, EEUU: J. W. Sons, Ed.

Nicolis, G., \& Prigogine, I. (2007). La estructura de lo complejo: en el camino hacia una nueva comprensión de las ciencias. México: Alianza.

Nonaka, I. (1991): The knowledge-creating company. Harvard Business Review, November-December, 96-104

PM Solutions. (2007). State of the PMO. CBP.

PMI. (2008). Guía de los fundamentos para la dirección de proyectos (guía del Pmbok) $4^{\circ}$ ed. Pennsylvania: Project Management Institute, Inc.

Prigogine, I. (1983). Tan solo una ilusión: una exploración del orden a partir del desorden. Barcelona: Tusquets.

Rivas, L. M. (2014). Conocimiento gerencial en empresas multinegocios Caso Suramericana S.A. Medellín, Colombia: Editorial EAFIT.

Rouse, W. (2000). Managing complexity: disease control as a complex adaptive system. Information. Systems Management, 2, 143-165.

Senge, P. (1990). The Fifth Discipline: The Art and Practice of the Learning Organization. London: Century. 
Skok, W. \& Kalmanovitchb, C. (2005). Evaluating the role and effectiveness of an intranet in facilitating knowledge management: a case study at surrey county council. Information and Management. 42(5), $731-744$.

Small, C. \& Sage, P. (2009). A Complex Adaptive Systems Based Enterprise Knowledge Sharing Model. International Journal of Enterprise Information Systems, 5(2), 217 - 236.

Smith, A. (2005). Complexity theory for organizational future studies. Foresight, Vol. 7 (3), 22-30.

Stacey, D. R. (1995). The science of complexity: An alternative perspective for strategic change processes. Strategic Management Journal, 16(6), 477-495.

Stacey, R. (2003). Organizations as complex responsive processes of relating. Journal of Innovative Management, 8(2), 27-39.

Stanislavsky, A. (2000). Memory effects and macroscopic manifestation of randomness. Phys. Rev, 61(5), 4752-4759.

Tetenbaum, T. (1998). Shifting paradigms: from Newton to chaos, Organizational Dynamics. 26(4) 21-32.

Watts, D., \& Strogatz, S. (1998). Collective dynamics of small world network. Nature, 42(5), 440-442. 
